\title{
PREÇO DE TRANSFERÊNCIA FISCAL EM EMPRESAS EXPORTADORAS BRASILEIRAS: UM ENFOQUE CONTINGENCIAL
}

\author{
Joice Denise Schäfer ${ }^{1}$, Valdirene Gasparetto, Luis Felipe Ferreira \\ Universidade Federal de Santa Catarina - UFSC, Santa Catarina, (Brasil)
}

\section{DETALHES DO ARTIGO}

\section{Histórico do Artigo:}

Recebido em: 24 dezembro 2018

Aceito: 15 julho 2019

Disponível online: 01 de agosto de 2019

Sistema de revisão "Double blind review"

\section{Editor Científico}

Ilan Avrichir

\section{Palavras-chaves:}

Preço de transferência fiscal

Fatores contingenciais

Empresas exportadoras.

\begin{abstract}
RESUMO
Este estudo tem como objetivo analisar os métodos de preço de transferência adotados por empresas exportadoras brasileiras, para fins fiscais, a partir de fatores contingenciais - ambiente, estratégia, porte, tecnologia e estrutura. O estudo caracteriza-se como descritivo, com abordagem qualitativa, realizado por meio de questionários aplicados a 90 empresas listadas no Catálogo de Exportadores Brasileiros. Os resultados evidenciam que, dentre as empresas que importam de vinculadas, o método mais utilizado é baseado no preço de revenda menos lucro (PRL), enquanto entre as empresas exportadoras é mais comumente empregado o método do custo de aquisição ou de produção mais tributos e lucro (CAP). Empresas menos verticalizadas, situadas em ambiente mais estável, centralizadas, de menor porte e que possuem nível tecnológico mais baixo tendem, em sua maioria, a empregar métodos similares em operações de importação e de exportação, possibilitando maior facilidade de controle e de comprovação do método empregado em casos de fiscalização.
\end{abstract}

\section{Introdução}

A internacionalização é uma opção para o crescimento das empresas, mas também uma necessidade frente a um mercado interno com altos níveis de concorrência e saturação comercial (Descals, Frasquet, \& Molina, 2011). As transferências entre empresas vinculadas, nas últimas décadas, vêm ganhando espaço neste cenário. Nos anos de 1960 as transferências compreendiam, em média, entre $20 \%$ e 33\% do total de transações internacionais (Lall, 1973), já nos anos de 1990 atingiram aproximadamente $40 \%$ das transações (Emmanuel, 1999) e nos anos 2000 o percentual chegou a $60 \%$ (Cool, Emmanuel, \& Jorissen, 2008).

Devido a estes aspectos de internacionalização empresarial, o lucro passou, cada vez mais, a ser distribuído entre unidades localizadas em diferentes países. Esta mudança, além de afetar o desempenho das unidades envolvidas, pode afetar o lucro global da organização, uma vez que os tributos cobrados sobre o lucro variam de um país para o outro. Nestas circunstâncias, o montante pelo qual se dá a transferência de bens ou serviços entre subsidiárias da mesma organização, denominado como preço de transferência, desempenha papel de destaque (Agana, Mohamed, \& Zamore, 2018).

Como os produtos transferidos não são negociados em mercados livres e abertos, podem desviar-se do valor de comercialização praticado entre empresas não relacionadas. Diante disso, visando proteger a base tributária e evitar a evasão fiscal, os países tentam, por meio de restrições legais, assegurar que os preços de transferência não estejam desproporcionalmente altos ou baixos (Giacomelli \& Aguiar, 2017). Neste sentido, a OECD (Organization for Economic Cooperation and Development) introduziu o princípio $a r m$ 's length, em que o preço praticado entre empresas vinculadas em transferências internacionais deve ser semelhante ao utilizado em transações com empresas independentes (OECD, 2010).

Com base no arm's length a OECD propôs, por meio de um guia de orientações (Guidelines), cinco métodos a serem adotados nas transações de 
importação e exportação entre empresas relacionadas. Assim como para fins gerenciais, para fins fiscais também não há um método de preço de transferência que assegure os melhores resultados para todas as empresas (Comerlato \& Procianoy, 1994; Schäfer, Petri, Gasparetto, \& Mattos, 2015). Assim, para definir o método a ser empregado é preciso analisar as características e objetivos da organização (Giacomelli \& Aguiar, 2017; Schafer, Gasparetto, Schnorremberger, \& Lunkes, 2017). Neste sentido, pesquisas anteriores têm estudado o processo de decisão do preço de transferência internacional, apoiando-se nas características organizacionais e ambientais das empresas, que conforme preconiza a Teoria Contingencial, tendem a influenciar os objetivos das organizações, interferindo também na seleção do método de preço de transferência.

Estudos sobre esta temática têm sido desenvolvidos desde a década de 1970 (Tang \& Chang, 1977; Tang, 1982; Borkowski, 1992; Chan \& Chow, 1997; 2001; Chan \& Lo, 2004; Klassen; Lisowsky, \& Mescall, 2017; Challoumis, 2018), os resultados, entretanto, divergem tanto sobre os motivos pelos quais determinado método de preço de transferência é selecionado em detrimento de outro, quanto sobre a interferência dos fatores ambientais e organizacionais na sua escolha. Este estudo se propõe, assim, a analisar os métodos de preço de transferência adotados por empresas exportadoras brasileiras, para fins fiscais, a partir de fatores contingenciais. Espera-se que a pesquisa possa contribuir com a prática ao fornecer informações que apoiem o planejamento tributário de empresas multinacionais, ou empresas que estão estudando a viabilidade de implementação de projetos com operações de transferências internacionais, sobre diferentes variáveis a serem consideradas na definição dos preços de transferência. Também se espera contribuição teórica, permitindo diminuir a lacuna de pesquisas que abordam de forma ampla tanto questões tributárias internacionais quanto os fatores contingenciais.

Os achados da pesquisa permitem identificar dois grupos de empresas, com diferentes características contingenciais e diferentes estratégias de preço de transferência, o que evidencia a relevância dos aspectos internos e ambientais, além de impostos e disputas com autoridades fiscais, no planejamento tributário das organizações.
A estrutura do artigo contempla, além da introdução apresentada, uma revisão teórica acerca do preço de transferência, métodos e determinações legais e uma revisão sobre os fatores contingenciais, procedimentos metodológicos empregados, resultados do estudo e discussões a respeito dos achados e, por fim, as conclusões da pesquisa.

\section{Referencial Teórico}

As transferências internacionais realizadas por empresas brasileiras estavam sujeitas apenas às regras de valoração aduaneira até o ano de 1996, quando foram inseridos, no direito brasileiro, métodos - baseados no princípio arm's length - para a determinação de um preço parâmetro (Silveira, 2007). Este preço de transferência é estabelecido com base nos métodos de preço de transferência constantes da legislação brasileira e serve para comparação com o preço que foi efetivamente praticado pelas empresas (Brasil, 2012a). Em 2012, a Lei no 12.715/12 (Brasil, 2012b) trouxe ajustes aos métodos fiscais introduzidos pela Lei no 9.430/96 (Brasil, 1996), anteriormente adotada.

Os métodos de preço de transferência fiscal são utilizados em operações de importação e exportação com pessoas vinculadas e em transações efetuadas com países de tributação favorecida (paraísos fiscais), com o objetivo de garantir que o preço praticado esteja dentro do que preconiza o princípio $a_{r m}$ s length (Brasil, 2012a). O princípio se refere, portanto, mais a um conceito do que a um valor fixo (Klassen; Lisowsky, \& Mescall, 2017). Além das empresas optarem por um método de preço de transferência que atenda ao princípio é necessário que mantenham registros e documentação das operações e dos preços de transferência praticados, para suportar as decisões da empresa junto às autoridades fiscais.

Caso o preço parâmetro calculado divirja em mais de $5 \%$ daquele que consta nos documentos de importação ou exportação, será exigido um ajuste no valor da diferença encontrada na base de cálculo dos impostos sobre o lucro -Imposto de Renda (IR) e da Contribuição Social sobre o Lucro Líquido (CSLL). Nas exportações, antes da aplicação dos métodos para determinação do preço parâmetro, as empresas devem, de acordo com a Instrução Normativa no 1.312 (Brasil, 2012a), analisar se o preço médio de venda dos bens, serviços ou direitos, praticado nas exportações durante o período de apuração da base de cálculo do IR e da CSLL, foi inferior a 90\% do preço médio praticado na venda dos bens, serviços ou direitos, idênticos ou similares, no mercado 
Preço de Transferência Fiscal em Empresas Exportadoras Brasileiras: Um Enfoque Contingencial

brasileiro, durante o mesmo período, em condições de pagamento semelhantes. Neste caso, as operações de exportação ficarão dispensadas do controle de preço de transferência.

\section{Métodos de preço de transferência brasileiros $x$ Guidelines da OECD}

As empresas localizadas no Brasil e que realizam transações significativas, de acordo com os requisitos apresentados no item anterior, com unidades vinculadas situadas em outros países, devem empregar o preço de transferência para calcular o preço parâmetro de suas transações e aplicar o preço adequado, a fim de assegurar o cumprimento do princípio arm's length. A legislação brasileira permite que as empresas optem por um dentre quatro métodos nas importações e por um dentre cinco nas exportações. Estes métodos de cálculo do preço de transferência estão relacionados de forma comparativa com os métodos estabelecidos pelo Guidelines da OECD (OECD, 2010), na Tabela 1.

Tabela 1 - Métodos de preço de transferência estabelecidos na legislação brasileira e OECD (2010)

\begin{tabular}{|l|l|}
\hline Métodos estabelecidos pela legislação brasileira & Métodos estabelecidos pela OECD \\
\hline Importação & Importação \\
\hline Método do preço independente comparado (PIC) & Comparable Uncontrolled Price Method (CUP) \\
\hline Método do preço de revenda menos lucro (PRL) & Resale Price Method (RPM) \\
\hline Método do custo de produção mais lucro (CPL) & Cost Plus Method (CPM) \\
\hline Método do preço sob cotação na importação (PCI) & - \\
\hline Exportação & Exportação \\
\hline Método do preço de venda nas exportações (PVEx) & Comparable Uncontrolled Price Method (CUP) \\
\hline $\begin{array}{l}\text { Método do preço de venda por atacado no país de } \\
\text { destino diminuído do lucro (PVA) }\end{array}$ & Resale Price Method (RPM) \\
\hline $\begin{array}{l}\text { Método do preço de venda a varejo no país de destino, } \\
\text { diminuído do lucro (PVV) }\end{array}$ & Resale Price Method (RPM) \\
\hline $\begin{array}{l}\text { Método do custo de aquisição ou de produção mais } \\
\text { tributos e lucro (CAP) }\end{array}$ & Cost Plus Method (CPM) \\
\hline Método do preço sob cotação na exportação (Pecex) & - \\
\hline
\end{tabular}

Fonte: Elaborado pelos autores (2015).

Em relação aos métodos para importação, brasileiros e da OECD, o PIC é um método similar ao CUP estabelecido pela OECD, e ambos sugerem que a transferência do bem ou serviço entre empresas relacionadas seja realizado pelo mesmo valor que seria feito caso a operação se realizasse entre empresas independentes, levando em consideração a qualidade e a quantidade de itens transferidos. $O$ PRL assemelha-se ao RPM, e ao optar por este método a empresa pratica na transferência o mesmo preço pelo qual o produto ou serviço final seria revendido a uma empresa independente, deduzido de uma margem bruta capaz de cobrir os gastos operacionais necessários para colocar o produto ou serviço em condições de venda; entretanto, as diretrizes da OECD (método RPM) permitem que a empresa estabeleça a margem a ser deduzida do valor final de revenda do bem ou serviço transferido, enquanto a lei brasileira (método $\mathrm{PRL}$ ) prevê margens fixas de dedução, que são de 20\%, 30\% e 40\%, variando de acordo com o setor de atuação da empresa. O CPL e o CPM partem do custo para formar o preço de transferência, e a este custo deve ser acrescido o mark up utilizado em vendas de produtos com margens similares entre empresas não relacionadas. Enquanto o $\mathrm{CPL}$ possui uma alíquota fixa a ser adicionada aos custos, de 20\%, no CPM a empresa deve identificar a alíquota a ser adicionada.

Dentre os métodos para exportação, o PVEx da legislação brasileira assemelha-se ao CUP proposto pela OECD, sendo basicamente o PIC aplicado às exportações. O PVA e o PVV são métodos similares ao $R P M$, porém o primeiro considera o valor final de revenda (reduzidos os impostos do país de destino) do item no atacado; e o segundo no varejo, de forma semelhante ao PRL usado na importação. Ambos possuem alíquotas a serem deduzidas do valor de venda fixadas na legislação, de $15 \%$ no caso da utilização do PVA e de $30 \%$ no uso do PVV. O CAP distingue-se do CPM, somente por ter acrescidos ao custo do produto ou serviço os impostos e contribuições cobrados no Brasil e uma margem de lucro de $15 \%$, fixada em lei.

O PCl, para as importações, e o Pecex, para as exportações, não possuem correspondentes nas diretrizes da OECD, pois aplicam-se somente a commodities e são baseados no valor de cotação diária das mesmas. Por outro lado, o Brasil não possui 
métodos de cálculo do preço de transferência com base no lucro das operações, como é o caso do Transactional Net Margin Method (TNMM) e do Transactional Profit Split Method (PSM) utilizados pelos países membros da OECD e demais que utilizam os métodos da OECD.

A legislação brasileira restringe a escolha do preço de transferência a ser praticado aos métodos mencionados na Tabela 1, no entanto, não há restrições adicionais relacionadas a características das organizações, em outras palavras as empresas podem optar pelo método que entenderem como o mais vantajoso para suas operações. Uma simulação da aplicação dos diferentes métodos, bem como os impactos nos resultados das organizações podem ser visualizados nos trabalhos de Giacomelli e Aguiar (2017) e Schafer et al. (2017).

\section{Preço de transferência e fatores contingenciais}

Devido às particularidades dos métodos, cabe a cada organização definir o melhor para precificar suas transferências, observando suas características e objetivos. Nessa linha, diversos autores buscaram identificar variáveis determinantes para a escolha do método de preço de transferência utilizado pelas empresas em diferentes países (Tang \& Chang, 1977; Tang, 1982; Borkowski, 1992; Chan \& Chow, 1997; 2001; Chan \& Lo, 2004; Klassen; Lisowsky, \& Mescall, 2017; Challoumis, 2018).

O foco destas pesquisas restringiu-se, basicamente, a variáveis ambientais, e nesse sentido, Scholes et al. (2014) e Klassen, Lisowsky \& Mescall (2017) afirmaram ser necessária uma visão mais ampla do planejamento tributário dentro da estratégia corporativa, sugerindo que um enfoque unidimensional poderia ignorar outras questões relevantes para a definição dos preços de transferência, relacionados, por exemplo, a descentralização ou custos (tributários ou não tributários). Neste sentido, o presente estudo busca analisar não apenas o ambiente externo à organização, mas também outros fatores contingenciais que podem influenciar a definição do preço de transferência.

Os fatores contingenciais podem ser entendidos como variáveis intervenientes, sejam internas ou externas à organização, que influenciam no processo de decisão devido às alterações que causam em determinadas características organizacionais (Donaldson, 1997). Ambiente (Burns \& Stalker, 1961;
Lawrence \& Lorsch, 1967; Thompson, 1976; Mintzberg, 1979; Gordon \& Naranayan, 1984), estratégia (Chandler, 1962; Eccles, 1983), porte da empresa (Mintzberg, 1979; Merchant, 1984), tecnologia (Woodward, 1965; Perrow, 1967; Thompson, 1976; Mintzberg, 1979; Gordon \& Naranayan, 1984) e estrutura (Burns \& Stalker, 1961; Chandler, 1962; Lawrence \& Lorsch, 1967; Perrow, 1967; Mintzberg, 1979; Merchant, 1984; Gordon \& Naranayan, 1984) são os fatores contingencias mais discutidos na literatura e, por este motivo, os fatores abordados nesta pesquisa.

O fator ambiente foi introduzido pelo estudo de Burns e Stalker (1961), que buscaram identificar a influência das mudanças tecnológicas e estabilidade ambiental sobre a estrutura das empresas. Lawrence e Lorsch (1967), Thompson (1976), Mintzberg (1979) e Gordon e Naranayan (1984) deram continuidade à pesquisa de Burns e Stalker (1961), analisando o efeito do ambiente sobre a estrutura organizacional, entretanto, considerando o fator ambiental sob diferentes enfoques. Nesta pesquisa, adotou-se a perspectiva mais recente, de Gorgon e Naranayan (1984), que analisaram o ambiente a partir da estabilidade considerando cinco variáveis explicativas: lançamento de novos produtos no mercado, previsibilidade dos competidores, previsibilidade das preferências dos clientes, novas descobertas (científicas ou tecnológicas) aplicadas ao setor, competitividade e restrições regulamentares. A variável competitividade foi analisada anteriormente nas pesquisas de Tang e Chang (1977) e Tang (1982) e as restrições legais foram avaliadas por Tang e Chang (1977), Tang (1982), Chan e Chow (1997) e Chan e Lo (2004).

No que se refere ao fator estratégia, Chandler (1962) defendeu que a estrutura da empresa decorre da estratégia adotada, sendo a estratégia analisada com base no grau de diversificação existente. Eccles (1983) avaliou a estratégia em duas dimensões: diversificação, a exemplo de Chandler, e integração vertical. Segundo Eccles (1983), com base nestas dimensões estratégicas as empresas podem ser divididas em coletiva, cooperativa, colaborativa ou competitiva. A divisão das empresas nestes grupos, de acordo com seu nível de diversificação e integração vertical, está demonstrada na Figura 1. 


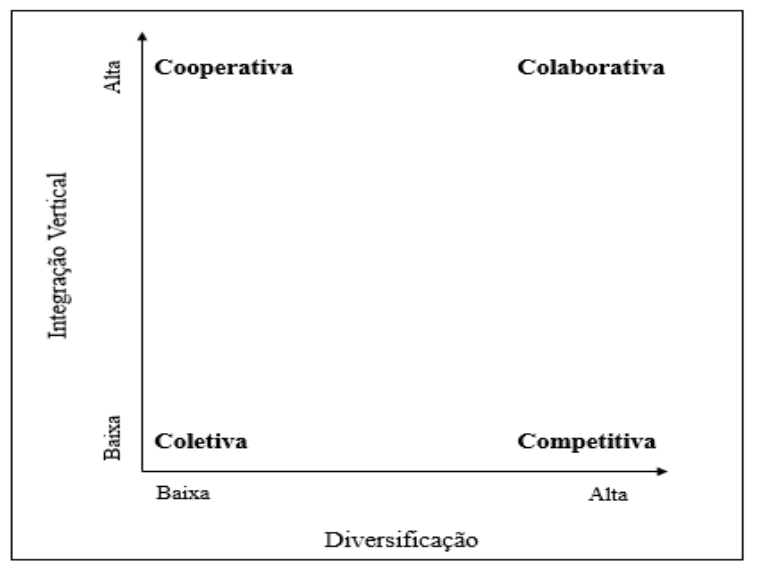

Figura 1. Tipos de empresa propostas por Eccles (1983) a partir das dimensões estratégicas

Fonte: Eccles, R. G. (1983). Control with fairness in transfer pricing. Harvard Business Review, 61(6), p. $149-161$.

As empresas classificadas por Eccles (1983) como coletivas não possuem processos sequenciais ou desenvolvimento de mais de um produto ou serviço, assim, tendem a não utilizar preço de transferência. As empresas cooperativas são aquelas em que há cooperação entre as unidades, e o preço com base no mercado tende a ser mais praticado, pois existe uma competição por lucros individuais entre as áreas, embora a cooperação seja necessária para a obtenção de um resultado global ótimo. O caso das empresas colaborativas é mais complexo, pois os resultados apresentam alta interdependência, assim não há um método de preço de transferência mais indicado (Eccles, 1983). Grau de diversificação e de integração são, portanto, as variáveis utilizadas neste estudo para a análise do fator estratégia.

O porte da empresa foi estudado por Mintzberg (1979) e Merchant (1984). Segundo os autores, na medida em que as empresas crescem, passam por processos de transição entre as diferentes estruturas: quanto maior o porte, maior também tende a ser o número de níveis hierárquicos e as exigências e normas de conduta. Nestas pesquisas, o porte foi medido pelo número de funcionários. Esse indicador, entretanto, com a inclusão de novas tecnologias e formas de contratação (terceirização de funcionários) pode distorcer o que se pretende medir. Neste sentido, optou-se por empregar neste estudo o faturamento como indicador base do tamanho da empresa. Esta variável também possui limitações, principalmente no que diz respeito à influência do setor em que as empresas operam, pois uma indústria transformadora, por exemplo, tende a ter maior valor agregado do que outros negócios.

A tecnologia foi inicialmente estudada por Woodward (1958; 1965), avaliando sua influência nos sistemas de produção e na estrutura organizacional, por meio da análise dos avanços tecnológicos e da automatização de processos produtivos. Thompson (1967) e Perrow (1967) expandiram o modelo de Woodward (1965). Thompson (1967) propôs um novo modelo composto por três tipos de tecnologia: tecnologia em elos de sequência, tecnologia mediadora e tecnologia intensiva. Perrow (1967) expandiu o conceito de tecnologia para além das máquinas e equipamentos utilizados na produção, incluindo o conhecimento necessário à transformação da matéria-prima em produto final. Em 1984, Gordon e Naranayan (1984), noutro estudo sobre o tema, focaram em sistema de gestão, considerando como variável de tecnologia o sistema de informação, que foi subdividido em informações externas, não-financeiras e ex ante.

As variáveis utilizadas nesta pesquisa referem-se a uso de hardware (máquinas e equipamentos) por parte das empresas estudadas, e o quão avançado ele é, como nas pesquisas de Woodward (1958; 1965), Thompson (1967) e Chan e Chow (2001), além disso, pela identificação da utilização de software e o conhecimento que os usuários possuem sobre a utilização dos mesmos (tanto do hardware, quanto do software), conforme a abordagem de Perrow (1967) e Gordon e Naranayan (1984). As variáveis qualidade dos materiais e qualificação das pessoas responsáveis pela produção são adicionadas a esta pesquisa, com o objetivo de atingir de forma mais ampla o conceito de Chenhall (2003) sobre as variáveis componentes do fator tecnologia.

Em diversos estudos observou-se que os fatores ambiente, estratégia, porte e tecnologia foram abordados concomitantemente a estrutura organizacional, estando esse fator presente em todas as pesquisas da abordagem contingencial, por ser influenciado pelos demais. Nas discussões sobre 
estrutura, diferentes classificações são propostas pelos pesquisadores. Woodward (1958; 1965), Burns e Stalker (1961) e Gordon e Naranayan (1984), por exemplo, a classificaram em mecanicista e orgânica; Chandler (1962), em divisional e funcional; Lawrence e Lorsch (1967) e Merchant (1984), em diversificação e integração; Thompson (1967) a analisou em seus diferentes níveis hierárquicos; Perrow (1967) considerou centralização e descentralização na tomada de decisões; Mintzberg (1979) adotou cinco classificações: estrutura simples, burocracia mecanizada, burocracia profissional, divisionalizada e adhocracia; e Eccles (1983) a dividiu em cooperativa, colaborativa, coletiva e competitiva, de acordo com os níveis de integração vertical e diversificação. Este estudo baseia-se na classificação das estruturas organizacionais em centralizadas e descentralizadas, conforme proposto por Perrow (1967) e replicado por Borkowski (1992).

\section{Procedimentos Metodológicos}

Para o desenvolvimento da pesquisa foram estudadas empresas internacionais, que transferem bens ou serviços entre subsidiárias localizadas em diferentes países. A partir das constatações de Cool, Emmanuel e Jorissen (2008), de que aproximadamente $60 \%$ do total das transações internacionais referem-se a transferências e não a operações de compra e venda, considerou-se um contexto adequado para a pesquisa as empresas listadas no Catálogo de Exportadores Brasileiros (2014). As empresas listadas no catálogo, que disponibilizaram na página algum email para contato foram contatadas e informadas a respeito da pesquisa. No mesmo email os autores solicitaram o contato do controller da empresa para futuro envio dos questionários. Houve um retorno de 259 empresas, disponibilizando os emails de seus controllers ou diretores financeiros. Dentre os 259 profissionais contatados, 90 (35\%) responderam à pesquisa, encaminhada por email no período de novembro a dezembro de 2015.

O questionário foi composto por três blocos, conforme descrito na Tabela 2. Os blocos 1 e 2 contemplaram escalas categóricas, enquanto o terceiro bloco foi composto por escalas intervalares de 0 a 10.

Tabela 2 - Estrutura do questionário

\begin{tabular}{|c|c|c|c|}
\hline Escala & Variáveis & Autores & $\begin{array}{c}N^{\mathbf{o}} \text { de } \\
\text { questões }\end{array}$ \\
\hline $\begin{array}{l}\text { Bloco 1: } \\
\text { Perfil dos } \\
\text { respondentes e } \\
\text { da empresa }\end{array}$ & $\begin{array}{l}\text { Função, sexo, tempo de atuação na empresa, tempo de } \\
\text { experiência na função, nível de escolaridade e área de } \\
\text { formação do respondente; e segmento de atuação da empresa. }\end{array}$ & Junqueira (2010) & 7 \\
\hline $\begin{array}{l}\text { Bloco 2: Preço } \\
\text { de transferência }\end{array}$ & $\begin{array}{l}\text { Existência de empresa vinculada; existência de transações } \\
\text { feitas com empresa vinculada; método de preço de } \\
\text { transferência fiscal utilizado; utilização do preço de } \\
\text { transferência gerencial; método de preço de transferência } \\
\text { gerencial utilizado; controles do preço de transferência } \\
\text { gerencial e fiscal. }\end{array}$ & $\begin{array}{l}\text { Chan e Chow (2001), } \\
\text { Borkowski (1990; 1992; } \\
\text { 1997) e Brasil (2010) }\end{array}$ & 9 \\
\hline $\begin{array}{l}\text { Bloco 3: Fatores } \\
\text { contingenciais }\end{array}$ & $\begin{array}{l}\text { Estratégia de integração vertical; estratégia de diversificação; } \\
\text { nível de centralização da tomada de decisão; nível de } \\
\text { formalização das funções; existência do manual do } \\
\text { colaborador; tamanho da empresa; nível de utilização de } \\
\text { softwares; nível tecnológico de hardwares; qualidade dos } \\
\text { materiais; e qualificação dos colaboradores; estabilidade } \\
\text { ambiental; existência de um preço de mercado para os bens } \\
\text { e/ou serviços transferidos; experiência da empresa com } \\
\text { auditorias. }\end{array}$ & $\begin{array}{c}\text { Eccles (1983); Gordon e } \\
\text { Naranayan (1984), } \\
\text { Borkowski (1990; 1992; } \\
\text { 1997) e Kouser } \text { et al. } \\
\text { (2012) }\end{array}$ & 8 \\
\hline
\end{tabular}

Antes do envio do questionário, foi realizado préteste com 3 empresas dentre as listadas no Catálogo de Exportadores Brasileiros, sendo uma integrante das 258 empresas que retornaram o contato feito por email ou site com o nome do controller, contador ou outro profissional que pudesse responder questões relacionadas ao preço de transferência e 2 empresas que se recusaram a participar da pesquisa, entretanto, aceitaram participar do pré-teste. A partir do pré-teste foram alteradas questões e termos para facilitar a compreensão das questões.

As respostas foram analisadas por meio de conglomerados (clusters), distribuídos de acordo com os fatores contingenciais ambiente, porte, estratégia, 
tecnologia e estrutura, classificando as empresas da pesquisa em grupos homogêneos dentro de cada um dos fatores para facilitar a compreensão dos diferentes grupos de empresas. Os clusters são analisados comparativamente na sequência, com o objetivo de identificar possíveis relações entre os fatores contingenciais e o preço de transferência utilizado.

Para o desenvolvimento desta pesquisa utilizouse, portanto, uma abordagem descritiva e qualitativa. O procedimento técnico utilizado para coleta dos dados foi o levantamento (survey), realizado por meio da aplicação de questionário.

\section{Resultados}

Das 90 empresas que responderam ao questionário, 23 (25,6\%) informaram possuir vinculadas no exterior, das quais 17 confirmaram realizar operações de importação de bens ou serviços destas unidades e 18 realizam operações de exportação. Três empresas afirmaram não realizar operações de importação ou exportação com as vinculadas, totalizando, desta forma, 20 empresas que utilizam o preço de transferência para fins fiscais. Destas 23, três empresas realizam apenas operações de exportação e duas de importação. Na sequência cada um dos fatores contingenciais é analisado qualitativamente no contexto das empresas estudadas, buscando identificar possíveis relações entre os fatores contingenciais e os métodos de preço de transferência fiscal praticados.

\section{Fator ambiente}

Para a análise das características contingenciais referentes ao fator ambiente, as empresas foram agrupadas com base nas variáveis utilizadas por Gordon e Naranayan (1984), especificamente da estabilidade das empresas no ambiente em que estão situadas, sendo o ambiente representado pela estabilidade ou dinamismo quanto a concorrência, mão-de-obra, insumo, tecnologia, restrições legais e clientes. Os clusters deste fator estão evidenciados na Tabela 3.

Tabela 3 - Clusters para o fator ambiente

\begin{tabular}{|c|c|c|c|}
\hline & Cluster & N. ${ }^{\circ}$ de empresas & $\begin{array}{c}\mathrm{N}^{\circ} \text { de empresas que } \\
\text { empregam o PT }\end{array}$ \\
\hline \multirow{4}{*}{ 竞 } & Dinâmico & 22 & 4 \\
\hline & Moderado & 46 & 11 \\
\hline & Estável & 22 & 5 \\
\hline & Total & 90 & 20 \\
\hline
\end{tabular}

Fonte: Dados da pesquisa (2015).

O primeiro cluster refere-se às empresas situadas em ambiente de maior dinamismo, sendo formado por 22 empresas que apresentam uma média de 48 pontos, dos 60 possíveis de serem atribuídos (quanto maior a pontuação, mais dinâmico o ambiente). O segundo grupo é formado por empresas que apresentam em média 37 pontos, e são classificadas como moderadas, uma vez que não apresentam baixa pontuação que as caracterizariam como estáveis, nem alta, que as classificariam como dinâmicas, e no último grupo estão concentradas as empresas que possuem média de apenas 21 pontos, apontando maior estabilidade das variáveis ambientais estudadas. Dentre cada um dos cluster, cerca de $20 \%$ das empresas informaram utilizar preço de transferência fiscal.

Ao analisar o preço de transferência fiscal aplicado às importações e exportações é possível notar diferença na utilização dos métodos praticados pelas empresas situadas em ambiente dinâmico e em ambiente estável, conforme Tabela 4, onde cada empresa da amostra foi representada por um número sequencial. 
Tabela 4 - Método do preço de transferência fiscal e ambiente da empresa

\begin{tabular}{|c|c|c|c|}
\hline Ambiente & Empresa & $\begin{array}{c}\text { Método de preço de } \\
\text { transferência (importação) } \\
\end{array}$ & $\begin{array}{c}\text { Método de preço de } \\
\text { transferência (exportação) }\end{array}$ \\
\hline \multirow{4}{*}{ Dinâmico } & 1 & PRL & CAP \\
\hline & 2 & PRL & CAP \\
\hline & 3 & PRL & CAP \\
\hline & 4 & & CAP \\
\hline \multirow{11}{*}{ Moderado } & 5 & PIC & PVEx \\
\hline & 6 & & PVV \\
\hline & 7 & PRL & PVA \\
\hline & 8 & PRL & $\mathrm{CAP}$ \\
\hline & 9 & PRL & \\
\hline & 10 & PRL & CAP \\
\hline & 11 & $\mathrm{CPL}$ & $\mathrm{CAP}$ \\
\hline & 12 & & PVEx \\
\hline & 13 & PRL & \\
\hline & 14 & PRL & CAP \\
\hline & 15 & PRL & PVA \\
\hline \multirow{5}{*}{ Estável } & 16 & PRL & $\mathrm{PVV}$ \\
\hline & 17 & PIC & CAP \\
\hline & 18 & PIC & PVEx \\
\hline & 19 & CPL & CAP \\
\hline & 20 & $\mathrm{CPL}$ & CAP \\
\hline
\end{tabular}

Fonte: Dados da pesquisa (2015).

A Tabela 4 mostra que no ambiente dinâmico as empresas aplicam diferentes bases metodológicas, sendo a metodologia baseada no preço de mercado nas importações (PRL - Preço de Revenda Menos Lucro) e a metodologia baseada nos custos na exportação (CAP Custo de Aquisição ou de Produção mais Tributos e Lucro), o que não é comum entre as empresas situadas em ambiente estável, sendo realizada por apenas uma (empresa 17) das cinco analisadas. As demais organizações do ambiente estável afirmaram utilizar o método de preço de transferência baseado nos mesmos critérios tanto nas importações como nas exportações, o que pode indicar o emprego de métodos com base na facilidade de controle, diferente do ambiente dinâmico onde o método empregado parece ser determinado como uma estratégia para a obtenção de melhores resultados por parte da empresa.

Dentre as empresas classificadas no segundo cluster (ambiente moderado), não foi possível notar um padrão na adoção do preço de transferência, assim como não houve uma classificação similar para as empresas que usam o preço de transferência para fins fiscais e gerenciais.

\section{Fator estratégia}

Para a análise do fator estratégia as empresas foram classificadas de acordo com o enquadramento proposto por Eccles (1983), em cooperativas, competitivas, colaborativas e coletivas, conforme evidenciado na Tabela 5.

Tabela 5 - Classificação das empresas no fator estratégia

\begin{tabular}{|c|c|c|c|}
\hline & Classificação & N. ${ }^{\circ}$ de empresas & $\begin{array}{l}\mathrm{N}^{\circ} \text { de empresas que } \\
\text { empregam o PT }\end{array}$ \\
\hline \multirow{4}{*}{ 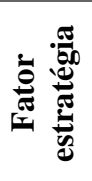 } & Cooperativas & 31 & 8 \\
\hline & Colaborativas & 12 & 2 \\
\hline & Competitivas & 4 & 0 \\
\hline & Coletivas & 43 & 10 \\
\hline
\end{tabular}

Fonte: Adaptado de Eccles (1983).

Segundo Eccles (1983), as empresas que não empregam preço de transferência, neste caso 70, deveriam apresentar baixa diversificação e integração vertical, ou seja, enquadrar-se na categoria de empresas coletivas, entretanto, nesta pesquisa somente 49\% (34 empresas) apontaram tais características, enquanto 23 (33\%) enquadraram-se como cooperativas, 10 (14\%) como colaborativas e 3 (4\%) como competitivas.
O fato das empresas da pesquisa apresentarem características estratégicas de organizações coletivas e cooperativas demonstra que poucas possuem diversificação produtiva. Ao analisar as empresas que utilizam preço de transferência para fins fiscais, estas mesmas características aparecem ainda com maior destaque, como pode ser confirmado na Figura 2. 


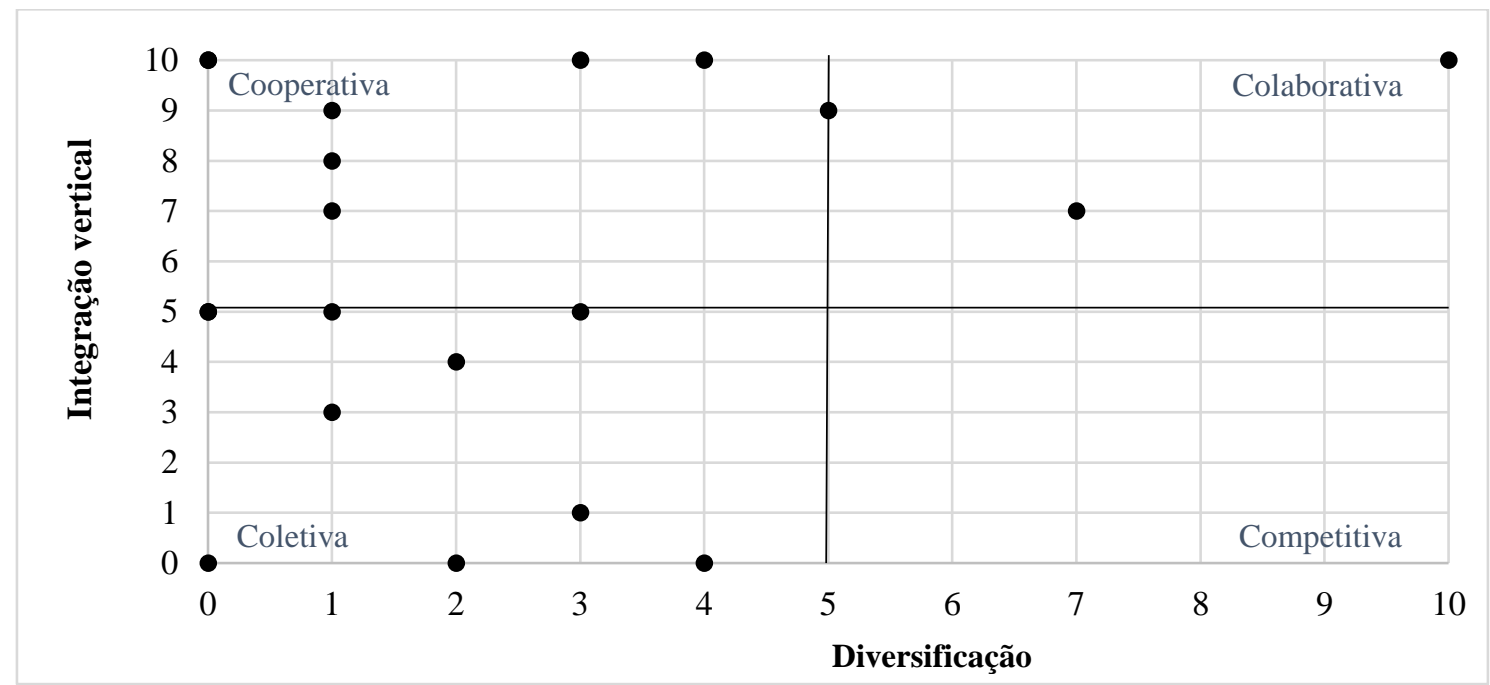

Figura 2. Classificação das empresas que utilizam preço de transferência fiscal de acordo com a diversificação e integração vertical

Fonte: Dados da pesquisa (2015).

A Figura 2 evidencia que apenas duas empresas que utilizam o preço de transferência para fins de exportação e/ ou importação, possuem um nível alto de diversificação, enquanto o nível de integração mostrouse bastante variável.

Ao analisar as empresas dentro da classificação proposta por Eccles (1983), portanto, a maior parte é agrupada em coletiva e cooperativa, sendo duas consideradas colaborativas e nenhuma enquadrada como competitiva.

As duas empresas colaborativas realizam tanto importação quanto exportação e utilizam preços com base no mercado (PIC - Preço Independente Comparado - e PVEx - Preço de Venda nas Exportações) para a determinação do preço a ser praticado nas transferências internacionais. Dentre as 10 empresas (duas empresas estão no ponto 5;0) coletivas, não há um padrão de utilização de método para a determinação do preço de transferência, sendo que das oito empresas importadoras, quatro utilizam PRL (Preço de Revenda menos Lucro), três empregam CPL (Custo de Produção mais Lucro) e uma usa PIC (Preço Independente Comparado).

No caso do preço de transferência fiscal não é possível comparar os métodos recomendados por Eccles (1983) para cada categoria de empresas, com o que é efetivamente praticado, uma vez que ao realizar transferências internacionais a empresa é obrigada a utilizar o preço de transferência, independente de possuir uma estratégia de baixa diversificação e integração vertical.

Ao considerar os métodos utilizados pelas empresas cooperativas, notou-se preferência pela utilização do PRL (Preço de Revenda menos Lucro) para a importação e baseado no custo para a exportação (CAP - Custo de Aquisição ou de Produção mais Tributos e Lucro). Das oito empresas (duas empresas estão no ponto 10;0), sete exportam e seis importam, sendo que na importação 100\% afirmaram utilizar o PRL (Preço de Revenda menos Lucro), e na exportação apenas uma afirmou não utilizar o CAP (Custo de Aquisição ou de Produção mais Tributos e Lucro), optando pelo PVV (Preço de Venda a Varejo no País de Destino, Diminuído do Lucro). Neste caso o método usado na maior parte das empresas para a exportação corresponde ao indicado por Eccles (1983), como o melhor a ser praticado em unidades de empresas com alta integração vertical e baixa diversificação, entretanto, a diferença de métodos adotados na importação e na exportação indica que, no caso do preço de transferência fiscal, o método sofre maior influência da estratégia fiscal adotada pelas empresas e não tem relação com os fatores contingenciais, foco desta pesquisa.

\section{Fator porte}

A classificação do fator porte foi realizada em cinco categorias, de acordo com a classificação do BNDES (2010), conforme evidenciado na Tabela 6. 
Tabela 6 - Classificação das empresas de acordo com o porte

\begin{tabular}{|c|c|c|c|}
\hline Faturamento & Classificação BNDES (2010) & N.o de empresas & $\begin{array}{c}\mathbf{N}^{\circ} \text { de empresas } \\
\text { que empregam } \\
\text { o PT } \\
\end{array}$ \\
\hline Microempresa & $\mathrm{R} \$ 0$-| R\$ 2,4 milhões & 3 & 1 \\
\hline Pequena empresa & $\mathrm{R} \$ 2,4$ milhões $-\mid \mathrm{R} \$ 16$ milhões & 13 & 3 \\
\hline Média empresa & $\mathrm{R} \$ 16$ milhões $-\mid \mathrm{R} \$ 90$ milhões & 35 & 6 \\
\hline Média-grande empresa & $\mathrm{R} \$ 90$ milhões -| R \$ 300 milhões & 21 & 4 \\
\hline Grande empresa & R\$ 300 milhões - & 8 & 3 \\
\hline \multicolumn{2}{|r|}{ Não informaram } & 10 & 3 \\
\hline \multicolumn{2}{|r|}{ Total } & 90 & 20 \\
\hline
\end{tabular}

Fonte: Adaptado do BNDES (2010)

Dez empresas pesquisadas optaram por não informar o valor do faturamento no ano de 2014. Dentre as demais a média do faturamento foi de $\mathrm{R} \$ 172.219 .741$, enquanto a mediana foi de $\mathrm{R} \$ 55.000 .000$, sendo que 16 (20\%) enquadram-se como micro ou pequena empresa. A diferença significativa entre média e mediana se dá por quatro outliers, sendo duas empresas que apresentam faturamento entre $\mathrm{R} \$ 900$ milhões e $\mathrm{R} \$ 1$ bilhão, outra entre $R \$ 1$ bilhão e $R \$ 2$ bilhões e a última acima de $R \$ 3$ bilhões. As empresas que utilizam o preço de transferência para fins fiscais apresentam faturamento entre $\mathrm{R} \$ 980 \mathrm{mil}$ e R\$ 3 bilhões, conforme evidenciado na Tabela 7.
Dentre os métodos utilizados na importação, demonstrados na Tabela 7, o CPL (Custos de Produção mais Lucro) é aplicado apenas nas transferências de micro e pequenas empresas. O PIC (Preço Independente Comparado) é utilizado por empresas de pequeno e médio porte, enquanto o PRL (Preço de Revenda menos Lucro) é usado por empresas de todos os tamanhos. Na exportação, nota-se que os métodos variam nas empresas de micro, pequeno e médio porte, entretanto todas as empresas de grande porte (com faturamento superior a $\mathrm{R} \$ 300$ milhões), que informaram o faturamento, optam pelo método baseado nos custos - CAP.

Tabela 7 - Faturamento das empresas que utilizam o preço de transferência fiscal

\begin{tabular}{cccc}
\hline Faturamento & Empresa & Método para importação & $\begin{array}{c}\text { Método para } \\
\text { exportação }\end{array}$ \\
\hline $\mathrm{R} \$ 980.000,00$ & 2 & PRL & CAP \\
\hline $\mathrm{R} \$ 2.800 .000,00$ & 5 & $\mathrm{PIC}$ & $\mathrm{PVV}$ \\
\hline $\mathrm{R} \$ 11.000 .000,00$ & 6 & $\mathrm{CIC}$ & $\mathrm{CAP}$ \\
\hline $\mathrm{R} \$ 12.000 .000,00$ & 17 & $\mathrm{PRL}$ & $\mathrm{PVA}$ \\
\hline $\mathrm{R} \$ 32.000 .000,00$ & 7 & $\mathrm{CPL}$ & $\mathrm{CAP}$ \\
\hline $\mathrm{R} \$ 56.000 .000,00$ & 11 & $\mathrm{CPL}$ & $\mathrm{CAP}$ \\
\hline $\mathrm{R} \$ 56.000 .000,00$ & 20 & $\mathrm{PRL}$ & $\mathrm{PVA}$ \\
\hline $\mathrm{R} \$ 60.000 .000,00$ & 9 & $\mathrm{CPL}$ & $\mathrm{CAP}$ \\
\hline $\mathrm{R} \$ 77.000 .000,00$ & 19 & $\mathrm{PRL}$ & $\mathrm{PVEx}$ \\
\hline $\mathrm{R} \$ 77.000 .000,00$ & 15 & $\mathrm{PRL}$ & $\mathrm{PIC}$ \\
\hline $\mathrm{R} \$ 117.000 .000,00$ & 8 & $\mathrm{PRL}$ & $\mathrm{CAP}$ \\
\hline $\mathrm{R} \$ 130.000 .000,00$ & 18 & $\mathrm{PRL}$ & $\mathrm{CAP}$ \\
\hline $\mathrm{R} \$ 132.825 .000,00$ & 13 & $\mathrm{PRL}$ & $\mathrm{CAP}$ \\
\hline $\mathrm{R} \$ 192.000 .000,00$ & 10 & & $\mathrm{PVV}$ \\
\hline $\mathrm{R} \$ 641.240 .086,75$ & 1 & $\mathrm{PRL}$ & $\mathrm{PVEx}$ \\
\hline $\mathrm{R} \$ 3.000 .000 .000,00$ & 3 & $\mathrm{PRL}$ & $\mathrm{CAP}$ \\
\hline não informou & 16 & $\mathrm{PRL}$ & \\
\hline não informou & 12 & & \\
\hline não informou & 14 & & \\
\hline
\end{tabular}

Fonte: Dados da pesquisa (2015).

Diante dos dados apresentados na Tabela 1, nota-se variação entre os métodos usados por empresas de pequeno porte (incluindo as microempresas), na importação ou na exportação. Entretanto, as empresas de médio porte (classificadas pelo BNDES [2010] como médiogrande) e de grande porte, exceto a empresa 18, optam pelo método baseado no mercado para importar e baseado no custo para exportar. Dentre as dez empresas de menor porte, esta prática foi identificada em apenas duas.

\section{Fator tecnologia}

Para a análise da tecnologia as empresas também foram divididas em três clusters. O primeiro refere-se às empresas com maior nível tecnológico, ou seja, aquelas que utilizam de forma intensa o ERP, automatizam o 
processo produtivo, usam as tecnologias mais avançadas no que diz respeito a máquinas e equipamentos e possuem alta qualidade nos insumos e qualificação adequada da mão-de-obra. O segundo cluster é composto por empresas que apresentam nível moderado das características citadas, enquanto o último agrupamento é composto por empresas que possuem nível baixo de tecnologia, em relação às variáveis pesquisadas. Esta divisão está evidenciada na Tabela 8.

Tabela 8 - Clusters para o fator tecnologia

\begin{tabular}{|c|c|c|c|}
\hline & Cluster & N.o de empresas & $\mathrm{N}^{0}$ de empresas que empregam o PT \\
\hline \multirow{4}{*}{ 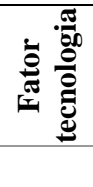 } & Alta & 59 & 15 \\
\hline & Moderada & 27 & 4 \\
\hline & Baixa & 4 & 1 \\
\hline & Total & 90 & 20 \\
\hline
\end{tabular}

Fonte: Dados da pesquisa (2015).

Nota-se, na Tabela 8, que apenas quatro respondentes indicaram que a empresa possui baixo nível tecnológico, enquanto aproximadamente 65\% das empresas apresentam alto nível tecnológico, o que se reflete também nas empresas que realizam transações internacionais. Na Tabela 9 está evidenciada a classificação das empresas de acordo com o método de preço de transferência utilizado nas importações e exportações.

Tabela 9 - Método do preço de transferência fiscal e fator tecnologia

\begin{tabular}{|c|c|c|c|}
\hline Tecnologia & Empresa & Método para importação & Método para exportação \\
\hline \multirow{15}{*}{ Alta } & 1 & PRL & CAP \\
\hline & 2 & PRL & CAP \\
\hline & 3 & PRL & CAP \\
\hline & 4 & & CAP \\
\hline & 5 & PIC & PVEx \\
\hline & 6 & & PVV \\
\hline & 7 & PRL & PVA \\
\hline & 8 & PRL & CAP \\
\hline & 9 & PRL & \\
\hline & 12 & & PVEx \\
\hline & 13 & PRL & \\
\hline & 14 & PRL & CAP \\
\hline & 15 & PRL & PVA \\
\hline & 16 & PRL & PVV \\
\hline & 17 & $\mathrm{PIC}$ & CAP \\
\hline \multirow{4}{*}{ Moderada } & 10 & PRL & CAP \\
\hline & 11 & $\mathrm{CPL}$ & CAP \\
\hline & 18 & PIC & PVEx \\
\hline & 20 & CPL & CAP \\
\hline Baixa & 19 & CPL & CAP \\
\hline
\end{tabular}

Fonte: Dados da pesquisa (2015).

O Tabela 9 evidencia que quatro empresas com tecnologia baixa e moderada tendem a utilizar métodos similares nas importações e exportações. Ao analisar conjuntamente o fator tecnologia (Tabela 9) e ambiente (Tabela 4), pode-se notar que as empresas classificadas no cluster 2 (tecnologia moderada) e 3 (baixa tecnologia) apresentam, também, dinamismo moderado (cluster 2 do fator ambiente) ou estabilidade no ambiente em que está situada (cluster 3 do fator ambiente). As empresas que apresentam alta tecnologia apresentam diferentes estratégias na adoção do preço de transferência. Observa-se ainda, na Tabela 8, que as empresas que realizam apenas importação ou apenas exportação também estão classificadas neste grupo.

\section{Fator estrutura}

Referente ao fator estrutura, identificou-se 22 empresas com alto nível de centralização, em que as decisões são altamente centradas na alta administração; 45 empresas descentralizadas, onde a tomada de decisão é atribuída a gestores de nível tático; e 23 empresas que afirmaram estar em nível intermediário de descentralização da tomada de decisão, conforme evidenciado na Tabela 10. 
Tabela 10 - Clusters para o fator estrutura

\begin{tabular}{|c|c|c|c|}
\hline & Cluster & N. ${ }^{\circ}$ de empresas & $\begin{array}{l}N^{\circ} \text { de empresas que } \\
\text { empregam o PT }\end{array}$ \\
\hline \multirow{4}{*}{ 灾 } & Descentralizadas & 45 & 9 \\
\hline & Moderada & 23 & 6 \\
\hline & Centralizadas & 22 & 5 \\
\hline & Total & 90 & 20 \\
\hline
\end{tabular}

Fonte: Dados da pesquisa (2015).

Os achados da pesquisa corroboram com Borkowski (1992) no que diz respeito à comparação entre o nível de centralização de empresas nacionais e internacionais. De acordo com a autora, empresas internacionais são mais centralizadas, o que pode ser comprovado a partir da média de centralização das organizações estudadas. Enquanto a média de centralização nas empresas nacionais foi de 4,6, nas empresas internacionais foi de 6,1, sendo as principais variações apontadas justamente na centralização da tomada de decisão quanto ao preço de venda e de transferência.

As empresas que utilizam preço de transferência representam um estrato similar, referente ao nível de descentralização, quando comparadas ao número de empresas totais.

Na Tabela 11 estão evidenciados os métodos de preço de transferência fiscal utilizados pelas empresas, de acordo com a estrutura.

Tabela 11 - Método do preço de transferência fiscal e fator estrutura

\begin{tabular}{|c|c|c|c|}
\hline Estrutura & Empresa & Método para importação & Método para exportação \\
\hline \multirow{9}{*}{ Descentralizada } & 1 & PRL & CAP \\
\hline & 2 & PRL & CAP \\
\hline & 9 & PRL & \\
\hline & 10 & PRL & CAP \\
\hline & 13 & PRL & \\
\hline & 15 & PRL & PVA \\
\hline & 17 & PIC & CAP \\
\hline & 18 & PIC & PVEx \\
\hline & 20 & $\mathrm{CPL}$ & CAP \\
\hline \multirow{6}{*}{ Moderada } & 6 & & PVV \\
\hline & 8 & PRL & CAP \\
\hline & 12 & & PVEx \\
\hline & 14 & PRL & CAP \\
\hline & 16 & PRL & PVV \\
\hline & 19 & CPL & CAP \\
\hline \multirow{5}{*}{ Centralizada } & 3 & PRL & CAP \\
\hline & 4 & & CAP \\
\hline & 5 & PIC & PVEx \\
\hline & 7 & PRL & PVA \\
\hline & 11 & CPL & CAP \\
\hline
\end{tabular}

Fonte: Dados da pesquisa (2015).

Dentre as quatro empresas centralizadas, que realizam operações de importação e exportação com empresas vinculadas, três utilizam métodos similares nas duas operações. Além da centralização, estas empresas apresentam um nível de estabilidade ambiental moderado e estão enquadradas como pequenas e médias empresas. A empresa três é a única dentre as empresas centralizadas a praticar métodos com bases diferentes nas operações de importação e exportação, e chama atenção por ser a empresa de maior porte dentro as pesquisadas e manter a tomada de decisão mais centralizada na alta administração para todas as variáveis analisadas. As demais empresas ( 6 de 7), que aplicam métodos de preço de transferência para importação baseados no mercado e com base nos custos para as exportações apresentam estrutura de moderada a descentralizada.

\section{Discussão dos Resultados}

De modo geral, as empresas pesquisadas percebem-se em um ambiente moderado, 
apresentam alto nível tecnológico e baixo nível de diversificação produtiva e são, em sua maioria, descentralizadas, e enquadram-se como empresas de médio e grande porte. Dentre as organizações respondentes, 20 informaram utilizar preço de transferência.

Em relação aos métodos de preço de transferência fiscal utilizados, o método PRL (preço de revenda menos lucro) foi apontado por $65 \%$ das empresas como o mais utilizado nas importações, e apenas uma empresa apontou utilizar o método que se baseia nos custos - CPL (custo de produção mais lucro). Já nas exportações, o método CAP (custo de aquisição ou de produção mais tributos e lucro) foi o mais utilizado, em $61 \%$ das empresas. A aplicação dos métodos específicos para operações com commodities não foi identificada em importações ou exportações.

Dentre as empresas da amostra que usam métodos baseados no mercado para importar e nos custos para exportar, a maior parte apresenta alta integração e baixa diversificação, alto nível tecnológico, com porte entre médio e grande, estrutura descentralizada, e estão situadas em ambiente dinâmico. As empresas menos verticalizadas, situadas em ambiente mais estável, mais centralizadas, de menor porte e que possuem nível tecnológico mais baixo informaram, em sua maioria, usar os mesmos métodos para importar e exportar. Estes achados podem ser justificados por uma diferenciação nos objetivos do primeiro grupo de empresas para o segundo.

O primeiro grupo é composto por empresas de médio ou grande porte, que tendem a realizar maior volume de transações internacionais do que as empresas de pequeno porte, situadas no segundo grupo. As operações de transferência internacionais tendem a ter maior representatividade nos resultados das organizações, logo exigem maior planejamento para a definição do método de preço de transferência a ser praticado. Em outras palavras, enquanto o segundo grupo opta por utilizar o mesmo preço de transferência para importações e exportações, devido à facilidade de controle e comprovação do método empregado em caso de fiscalização, as empresas do primeiro grupo definem os métodos de acordo com a perspectiva de melhores resultados de economia fiscal.

Além disso, as empresas do primeiro grupo apresentam maior nível de descentralização, exigindo maiores controles gerenciais e melhores sistemas de informação (o que pode ser comprovado pelo alto nível tecnológico informado por estas organizações), enquanto as empresas do segundo grupo, mais centralizadas, dispõem de sistemas de informações mais simples, enfrentando, assim, maior dificuldade para controlar diferentes metodologias de preço de transferências nas entradas e nas saídas.

Os resultados desta pesquisa evidenciam, portanto, que a definição da metodologia de preço de transferência a ser praticado pelas empresas é complexa e está associada a diferentes fatores e por este motivo essas escolhas não devem ser analisadas de forma unidimensional (Klassen; Lisowsky, \& Mescall, 2017). Com a complexidade associada aos preços de transferência, é importante que o método seja definido mediante um planejamento tributário realizado juntamente com o planejamento estratégico (Giacomelli \& Aguiar, 2017), que indicará o método que melhor se adequa às condições de cada empresa. Esta análise deve englobar tanto a redução de impostos ou disputas com autoridades fiscais, como as condições internas da empresa para controlar as transferência, a representatividade destas para os resultados da organização e custos relacionados, os objetivos da empresa mediante tais operações, entre outros.

\section{Conclusões}

Este trabalho teve como objetivo analisar os métodos de preço de transferência adotados por empresas exportadoras brasileiras, para fins fiscais, a partir de fatores contingenciais. A pesquisa foi aplicada a 259 empresas brasileiras listadas no Catálogo de Empresas Exportadoras disponibilizado pela Confederação Nacional das Indústrias. Um total de 90 empresas responderam o questionário da pesquisa, sendo que delas 20 informaram utilizar o preço de transferência para fins fiscais. Dentre estas empresas foi possível caracterizar dois grupos.

O primeiro grupo é composto por empresas com estratégia cooperativa, alto nível tecnológico, com porte entre médio e grande, estrutura descentralizada e situadas em ambiente dinâmico. Estas empresas tendem a adotar diferentes metodologias nas operações de importação e exportação entre subsidiárias, uma vez que tendem a preocupar-se mais com o impacto tributário das transações no resultado e competitividade, e menos com os controles fiscais, uma vez que tendem a possuir sistemas de controles aprimorados. 
O segundo grupo de empresas, menos verticalizadas, situadas em ambiente mais estável, mais centralizadas, de menor porte e que possuem nível tecnológico mais baixo informaram, em sua maioria, usar os mesmos métodos, para importação e exportação. Estas organizações podem estar voltadas à aplicação dos mesmos métodos de preço de transferência nas importações e exportações para maior facilidade de controle e de comprovação do método empregado em casos de fiscalização. Os achados demonstram a relevância dos fatores contingenciais para o planejamento tributário das organizações.

Com relação às limitações da pesquisa, destaca-se que: i) os dados coletados baseiam-se na percepção dos respondentes do questionário utilizado na pesquisa, desta forma, é possível que a aplicação do mesmo questionário a outros profissionais da

\section{Referências}

Agana, J. A., Mohammed, A. K., \& Zamore, S. (2018). International transfer pricing and income shifting in developing countries: evidence from Ghana. International Journal of Emerging Markets, 13(5), 1132-1153.

Bertolucci, A. V. (2001). "Uma contribuição ao estudo da incidência dos custos de conformidade às leis $e$ disposições tributárias: um panorama mundial e pesquisa dos custos das companhias de capital aberto no Brasil". Tese de Doutorado. Universidade de São Paulo - USP.

BNDES. (2010). "Porte de empresa". Rio de Janeiro. Disponível em: <http://www.bndes.gov.br/SiteBNDES/bndes/bndes_pt/l nstitucional/Apoio_Financeiro/porte.html>. Acesso em: 20 dez. 2015.

Borkowski, S. C. (1992) "Choosing a transfer pricing method: a study of the domestic and international decision-making process".Journal of international accounting, auditing and taxation, vol. 1, num. 1, pp. 3349. https://doi.org/10.1016/1061-9518(92)90005-Z

Brasil (1996). “Lei no 9.430 de 27 de dezembro de 1996". Dispõe sobre a legislação tributária federal, as contribuições para a seguridade social, o processo administrativo de consulta e dá outras providências.

Brasil a (2012). "Lei no 12.715 de 17 de setembro de 2012". 2012a.

Brasil b (2012). "Instrução Normativa 1.312 de 28 de dezembro de 2012". Dispõe sobre os preços a serem praticados nas operações de compra e de venda de bens, serviços ou direitos efetuadas por pessoa física ou jurídica residente ou domiciliada no Brasil, com pessoa física ou jurídica residente ou domiciliada no exterior, consideradas vinculadas. 2012b. empresa gerasse resultados divergentes; ii) as proxys utilizadas para representar as variáveis foram tomadas a partir de estudos anteriores e de conceitos obtidos na literatura, entretanto, há outras que poderiam ser utilizadas; e iii) houve dificuldade de acesso às empresas que realizam operações de transferência com vinculadas localizadas em outro país, empregando o preço de transferência, pois este dado não está disponível ao público no Brasil.

Recomenda-se que em estudos futuros sejam investigados, além dos fatores contingenciais, aspectos referentes a posição acionária que, conforme Comerlato e Procianoy (1994), podem gerar conflitos entre controladores e acionistas minoritários devido à possível transferência de riqueza para os primeiros, bem como fatores comportamentais relacionados aos responsáveis pela tomada de decisões.

Burns, T., \& Stalker, G. (1961). "The management of innovation". Tavistock, London.

Chan, K. H., \& Chow, L. (1997) International transfer pricing for business operations in China: Inducements, regulation and practice. Journal of Business Finance \& Accounting, vol. 24, num. 9-10, pp. 1269-1289. 10.1111/1468-5957.t01-1-00162

Chan, K. H., \& Chow, L. (2001). "Corporate environments and international transfer pricing: an empirical study of China in a developing economy framework". Accounting and Business Research, vol. 31, num. 2, pp. 103-118. http://dx.doi.org/10.1080/00014788.2001.9729605

Chan, K. H., \& Lo, A. (2004). "The influence of management perception of environmental variables on the choice of international transfer-pricing methods". The International Journal of Accounting, vol. 39, num. 1, pp. 93110. https://doi.org/10.1016/j.intacc.2003.12.004

Chandler, A. (1962). "Strategy and structure". MIT Press, Cambridge.

Chenhall, R. H. (2003). "Management control systems design within its organizational context: findings from contingency-based research and directions for the future". Accounting, Organizations and Society, vol. 28, num. 2, pp. 127-168. https://doi.org/10.1016/S0361-3682(01)00027-7

Cool, M., Emmanuel, C., \& Jorissen, A. (2008). "Management control in the transfer pricing tax compliant multinational enterprise". Accounting, Organizations and Society, vol. 33, num. 6, pp. 603-628. https://doi.org/10.1016/j.aos.2007.05.004.

Challoumis, C. (2018). Methods of Controlled Transactions and the Behavior of Companies According to the Public and Tax Policy. Economics, 6(1), 33-43. 
Descals, A. M., Frasquet, M., \& Molina, M. E. R. (2011). Magnitud e intensidad de la internacionalización de la gran distribución minorista: una comparativa internacional. Innovar, vol. 21, num. 39, pp. 53-66.

Donaldson, L. (1997). "The normal science of structural contingency theory". In: Clegg, S. R., Hardy, C., \& Nord, W. R. Handbook of organizations studies. Sage, London, pp. 57-76.

Eccles, R. G. (1983). Control with fairness in transfer pricing. Harvard Business Review, 61(6), p. 149-161.

Emmanuel, C. R. (1999). Income Shifting and International Transfer Pricing: A Three-Country Example. Abacus, 35(3), p. 252-267.

Giacomelli, J. G. C., \& de Aguiar, H. (2017). Preço de transferência e seus impactos contábeis e fiscais dentro de uma empresa de filtros automobilísticos na importação e exportação de mercadorias. Revista Catarinense da Ciência Contábil, 16(49).

Gordon, L. A., \& Narayanan, V. K. (1984). Management accounting systems perceived environmental uncertainly and organization structure: an empirical investigation. Accounting, Organizations and Society, 9(1), p. 33-47.

Klassen, K. J., Lisowsky, P., \& Mescall, D. (2017). Transfer pricing: Strategies, practices, and tax minimization. Contemporary Accounting Research, 34(1), 455-493.

Lall, S. (1973) Transfer-pricing by multinational manufacturing firms. Oxford Bulletin of Economics and Statistics, 35(3), p. 173-195.

Lawrence, P. R., \& Lorsch, J. W. (1967) Organization and environment: managing differentiation and integration. Boston: Harvard Press.

Merchant, K. (1984). Influences on departmental budgeting: an empirical examination of a contingency model. Accounting, Organization and Society, 9(3), p. 291307.

Mintzberg, H. (1979). The structuring of organizations: a synthesis of the research. Englewood Cliffs, NJ: PrenticeHall.

Morgan, G. (2010). Imagens da organização. São Paulo: Atlas.

OCDE. (2010). OECD transfer pricing Guidelines for multinational enterprises and tax administrations. OCDE: Paris.
Perrow, C. (1967). A framework for the comparative analysis of organizations. American sociological review, $\mathrm{p}$. 194-208.

Porter, M. E. Estratégia competitiva. Rio de Janeiro: Campus, 1996.

Procianoy, J. L., \& Comerlato, G. M. B. (1994). A transferência de resultados entre empresas de capital aberto de um mesmo grupo econômico. Revista de Administra\&ccdeil; ão da Universidade de São Paulo, 29(2).

Schäfer, J. D., Petri, S. M., Gasparetto, V., \& dos Santos Mattos, L. (2015). Preços de transferência: uma análise bibliométrica da literatura internacional. Internext, 10(3), 71-85.

Schäfer, J. D., Gasparetto, V., Lunkes, R. J., \& Schnorrenberger, D. (2018). Preço de transferência: legislação dos países da América do Sul e implicações nas importações de bens e serviços. Revista de Contabilidade do Mestrado em Ciências Contábeis da UERJ, 22(2), 3-19.

Silveira, R. M. (2007). O controle de preços de transferência nas exportações. In: Borges, A. S., Fernandes, E. C., \& Peixoto, M. M. Manual dos preços de transferência no Brasil - celebração dos 10 anos da vigência da lei, p. 107159. São Paulo: MP Editora.

Solilová, V. (2014). Practical application of Art. 9 OECD model convention: the Czech Republic. Acta Universitatis Agriculturae et Silviculturae Mendelianae Brunensis, 62(2), p. $397-403$.

Tang, R. Y. W. (1982). Environmental variables of multinational transfer pricing: A UK perspective. Journal of Business Finance \& Accounting, 9(2), p. 179-189.

Tang, R. Y. W., \& Chan, K. H. (1979). Environmental Variables of International Transfer Pricing: A Japan-United States Comparison. Abacus, 15(1), p. 3-12.

Thompson, J. D. (1967). Organizations in action. New York: McGraw Hill.

Warren, C. S.; Reeve, J. M.; \& Fess, P. E. (2008). Contabilidade Gerencial.2. ed. São Paulo: Thomson.

Woodward, J. (1965). Industrial organization: theory and practice. London: Oxford Unity Press.

Woodward, J. (1958). Management and Technology. London: Her Majesty's Stationery Office. 
- Joice Denise Schäfer - Universidade Federal de Santa Catarina - UFSC, Santa Catarina, (Brasil). E-mail: schafer.joice@gmail.com Orcid id: https://orcid.org/0000-0001-6779-1079

- Valdirene Gasparetto - Universidade Federal de Santa Catarina - UFSC, Santa Catarina, (Brasil). E-mail: valdirenegasparetto@gmail.com Orcid id: https://orcid.org/0000-0002-2825-4067

- Luis Felipe Ferreira - Universidade Federal de Santa Catarina - UFSC, Santa Catarina, (Brasil). E-mail: luiz.felipe@ufsc.br Orcid id: https://orcid.org/0000-0003-4105-1560

\section{FISCAL TRANSFER PRICE IN BRAZILIAN EXPORTING COMPANIES: A CONTINGENCIAL APPROACH}

Joice Denise Schäfer, Valdirene Gasparetto, Luis Felipe Ferreira

Universidade Federal de Santa Catarina - UFSC, Santa Catarina, (Brasil)

\section{ARTICLE DETAILS}

\section{Article history:}

Received: 24 December 2018

Accepted: 15 July 2019

Available online August: 1th 2019

Double Blind Review System

Scientific Editor

Ilan Avrichir

\section{Keywords}

Fiscal transfer price

Contingency factors

Exporting companies

\begin{abstract}
This article aims to investigate the transfer pricing methods adopted by Brazilian exporting companies for fiscal purposes based on contingency factors - environment, strategy, size, technology and structure. The study is characterized as descriptive, with a quantitative approach, performed through questionnaires applied to 90 companies listed in the Brazilian Exporters Catalog. The results show that, among the companies that import linked products, the most used method is based on the price of resale minus profit ( $P R L)$, while among the exporting companies the method of cost of acquisition or production plus taxes and profit (CAP) is the most commonly employed. Less-verticalized companies, located in a more stable, centralized, smaller, and lower technological level tend to use similar methods in import and export operations, making it easier to control and prove the method employed in cases of supervision.
\end{abstract}

Para citar este artigo:

Schäfer, J., Gasparetto, V., \& Ferreira, L. (2019). Preço de Transferência Fiscal em Empresas Exportadoras Brasileiras: Um Enfoque Contingencial. Internext, 14(3), 235-250. doi:http://dx.doi.org/10.18568/internext.v14i3.503 